\title{
Miniature Integrated Spectrometer Fabricated on a Silicon-on-Insulator Substrate
}

\author{
Joost Brouckaert, Gunther Roelkens, Shankar Selvaraja, Wim Bogaerts, Pieter Dumon, Steven Verstuyft, Zon- \\ Qiang Yu, Dries Van Thourhout and Roel Baets \\ Ghent University - IMEC, Department of Information Technology (INTEC), Sint-Pietersnieuwstraat 41, 9000 Gent, Belgium \\ Joost.Brouckaert@intec.UGent.be
}

\begin{abstract}
We demonstrate a compact and cost-effective 30-channel integrated spectrometer fabricated on a nanophotonic silicon-on-insulator (SOI) platform. The spectrometer consists of a planar concave grating (PCG) demultiplexer with heterogeneously integrated III-V photodetectors. The device has a resolution of $3.2 \mathrm{~nm}$ and a small footprint of $\sim 2 \mathrm{~mm}^{2}$. The photodetectors have a dark current of $\sim 3 \mathrm{nA}$ at $5 \mathrm{~V}$ bias and a responsivity of $1 \mathrm{~A} / \mathrm{W}$. The on-chip loss for the central PCG channels is $3 \mathrm{~dB}$, resulting in an on-chip responsivity of $0.5 \mathrm{~A} / \mathrm{W}$.
\end{abstract}

\section{INTRODUCTION}

$\mathrm{P}$ LANAR waveguide spectrograph type (de-)multiplexers like arrayed waveguide gratings (AWGs) and planar concave gratings (PCGs) are essential components in WDM telecommunication networks and with increasing resolution and channel count, these devices could also find application in integrated microspectrometers. We previously demonstrated that the nanophotonic silicon-on-insulator (SOI) platform is a very interesting candidate for the fabrication of very compact, low-cost and high performance PCGs [1].

In this letter, we demonstrate a microspectrometer consisting of a PCG demultiplexer fabricated on an SOI substrate with heterogeneously integrated InGaAs photodetectors. These photodetectors are integrated by means of bonding unprocessed III-V dies (epitaxial layers down) onto the processed SOI wafer using DVS-BCB as an intermediate adhesive layer. After substrate removal, the detectors can be fabricated on a wafer scale and lithographically aligned to the underlying SOI waveguides [2]. This integration technique, in combination with the fabrication of the passive circuitry on low-cost SOI wafers using CMOS compatible processes could result in a compact, highly integrated and low-cost miniature photospectrometer.

\section{DESIGN AND FABRICATION}

The heart of the spectrometer is a 30-channel planar concave grating (PCG) demultiplexer (Fig 1.). It is fabricated on a $200 \mathrm{~mm}$ SOI wafer with a $220 \mathrm{~nm}$ thick Si top layer using

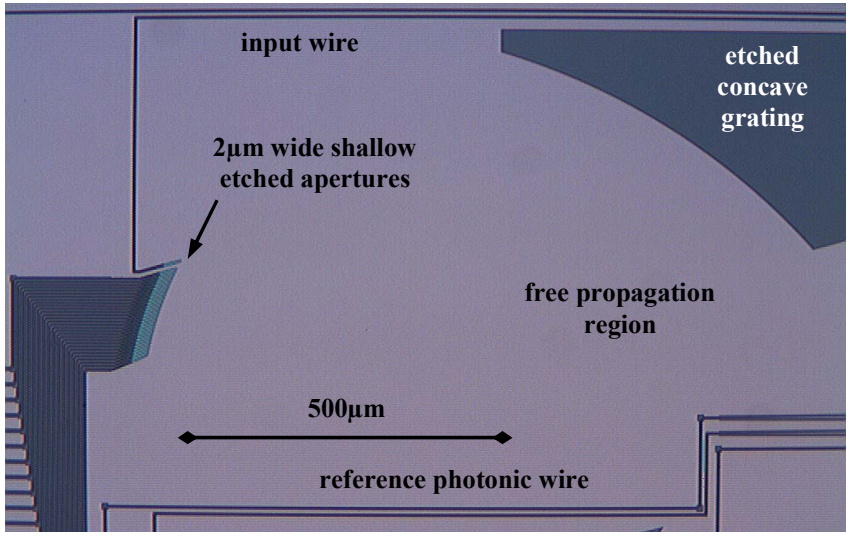

Fig. 1. Microscope picture of the 30-channel PCG demultiplexer.

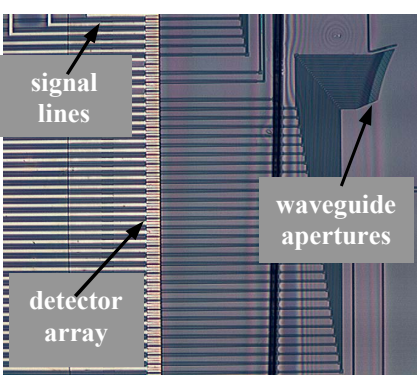

(a)

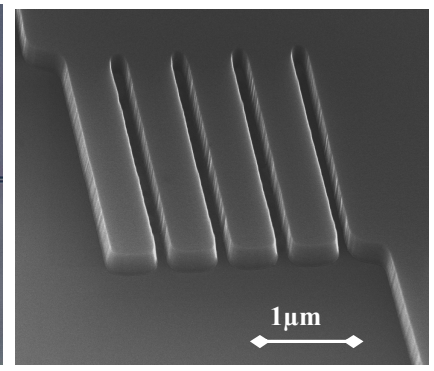

(b)
Fig. 2(a) Picture of the detector array processed on the output waveguides of the PCG demultiplexer and (b), a SEM picture of a DBR grating facet.

$193 \mathrm{~nm}$ deep-UV lithography in combination with ICP-RIE etching [3]. The design is based on the Rowland geometry: the input and output waveguides are positioned on a circle with a radius of $554 \mu \mathrm{m}$ and the curved grating sits on an $1108 \mu \mathrm{m}$ radius circle. The order of diffraction is 10 and the entrance and exit waveguides are $2 \mu \mathrm{m}$ wide with a spacing of $5 \mu \mathrm{m}$ between the centers of the output waveguides along the Rowland circle. These waveguides are shallowly etched (70nm deep) and a double adiabatic taper is used to convert to deeply etched $500 \mathrm{~nm}$ wide photonic wire waveguides. To avoid excessive reflection loss at the grating facets, we replaced the 141 flat facets with second order distributed Bragg reflector (DBR) facets [4] (Figure 2b). This method 


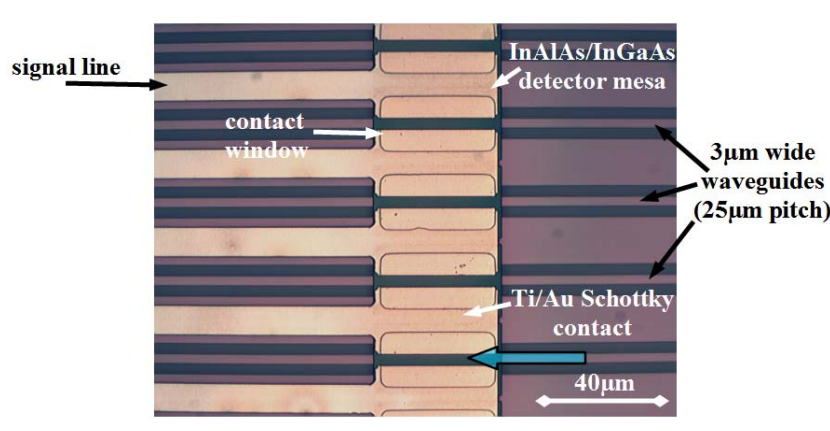

Fig. 3. Detailed top-view picture of the MSM photodetectors on top of $3 \mu \mathrm{m}$ wide SOI waveguides.

allows reducing the on-chip loss without the need of extra processing [4].

Evanescently coupled InGaAs/InAlAs metalsemiconductor-metal (MSM) photodetectors were integrated on top of the 30 output waveguides (Fig 2a). To obtain efficient coupling, the DVS-BCB bonding layer between the SOI waveguides and the MSM detectors is only $130 \mathrm{~nm}$ thick. Processing, simulation and measurements of these photodetectors are described elsewhere [2]. Detectors with a length of $40 \mu \mathrm{m}$ have a responsivity of $1 \mathrm{~A} / \mathrm{W}$ at $1.55 \mu \mathrm{m}$ and a dark current of $\sim 3 \mathrm{nA}$ at $5 \mathrm{~V}$ bias. The detector pitch is $25 \mu \mathrm{m}$ as can be seen in figure 3 . The size of the spectrometer including photodetectors, but excluding electrical probe pads is $\sim 2 \mathrm{~mm}^{2}$.

\section{MEASUREMENT RESULTS}

Light was coupled into the SOI chip using fiber couplers [3]. These are shallowly etched 1-D gratings which allow coupling light from a single mode fiber into the nanophotonic waveguides. Figure 4 shows the photocurrent spectrum of both the spectrometer and a detector which is processed on top of a reference waveguide. As the quantum efficiency of the detectors is almost constant from 1.5 to $1.6 \mu \mathrm{m}$, the spectrum of the reference detector is mainly determined by the fiber coupler transmission. These couplers have an estimated minimal coupling loss of $6 \mathrm{~dB}$ at a wavelength of $1590 \mathrm{~nm}$ and a $3 \mathrm{~dB}$ bandwidth of $65 \mathrm{~nm}$. The on-chip loss (in respect to the reference detector) of the PCG ranges from $3 \mathrm{~dB}$ for the central channels to $5 \mathrm{~dB}$ for the longest wavelength channel as can be seen in figure 4. The power budget can be calculated as follows. By fine-tuning the fiber coupler, it is possible to make the central PCG wavelength and the maximum fiber coupler transmission coincide at $1.55 \mu \mathrm{m}$. The total loss for the central channels is about $6 \mathrm{~dB}$ (fiber coupler loss) $+3 \mathrm{~dB}$ (on-chip PCG loss). Waveguide loss can be neglected. Taking into account a detector responsivity of $1 \mathrm{~A} / \mathrm{W}$ at $1.55 \mu \mathrm{m}$, the total responsivity for the central channels is $\sim 0.1 \mathrm{~A} / \mathrm{W}$. Due to the simultaneous processing of the detectors, there is no major variation of both the dark current and the responsivity for different detectors as can be seen in figure 5 .

The near-channel optical crosstalk varies between $-10 \mathrm{~dB}$ and $-18 \mathrm{~dB}$. To assess the longer range extinction ratio between the different spectrometer channels, we plotted the

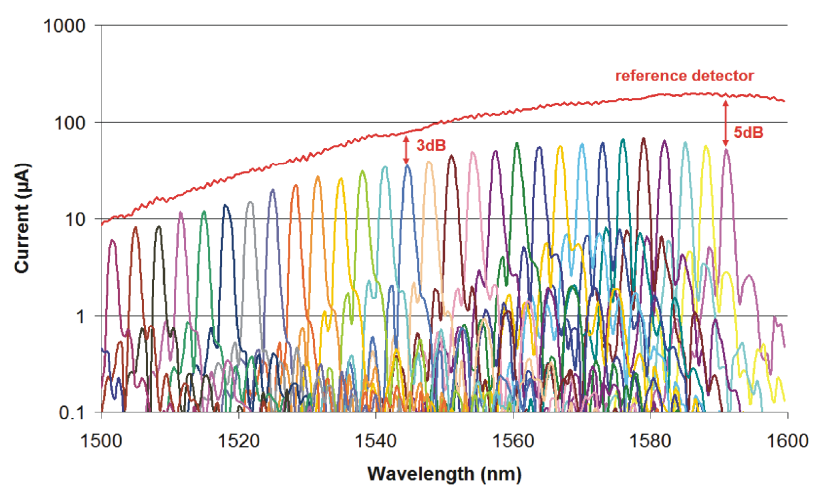

Fig. 4. Photocurrent spectrum of the 30-channel spectrometer and reference photodetector (red line) for TE polarized light. Only the sidelobes of the first channel (at $\lambda=1499$ ) are visible.

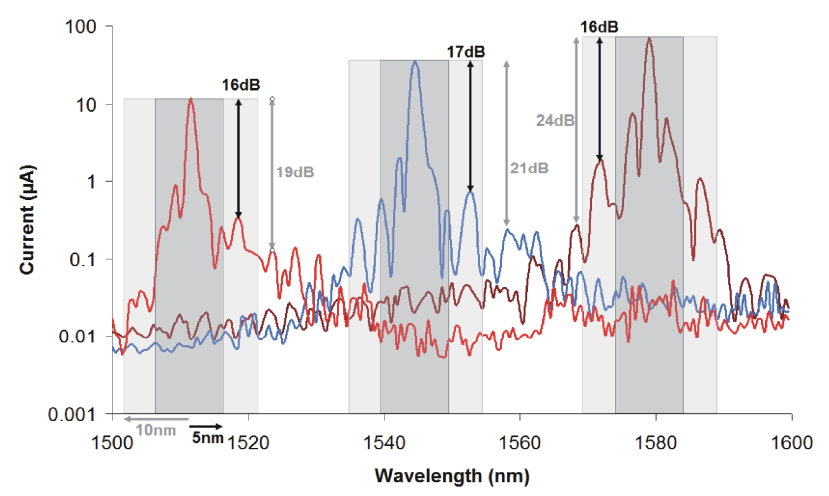

Fig. 5. Photocurrent spectrum and optical extinction ratio (both short and longer range) of channel 5, 15 and 26.

photocurrent of channels 5, 15 and 26 as can be seen in Figure 5 . The extinction ratio outside a $5 \mathrm{~nm}$ range of each channel is better than $15 \mathrm{~dB}$, and $>20 \mathrm{~dB}$ outside a $10 \mathrm{~nm}$ range.

\section{CONCLUSIONS}

We demonstrated a very compact $\left(2 \mathrm{~mm}^{2}\right)$ near-infrared 30channel spectrometer based on the heterogeneous integration of III-V photodetectors on SOI PCGs. As these photodetectors can be processed on a wafer scale on cheap and high quality SOI substrates, it opens the way for low-cost miniature spectrometers that can be mass-fabricated.

\section{ACKNOWLEDGMENT}

This work was supported in part by the IWT-SBO epSOC project.

\section{REFERENCES}

[1] J. Brouckaert et al., "Planar Concave Grating Demultiplexer Fabricated on a Nanophotonic Silicon-on-Insulator Platform," Journal of Lightwave Technology, vol. 25, pp. 1269-1275, 2007.

[2] J. Brouckaert et al., "Compact InAlAs/InGaAs Metal-SemiconductorMetal Photodetectors Integrated on Silicon-on-Insulator Waveguides," Photonics Technology Letters, vol. 19, pp. 1484-1486, 2007.

[3] W. Bogaerts et al., "Basic structures for photonic integrated circuits in Silicon-on-insulator," Optics Express, vol. 12, pp. 1583-1591, 2004.

[4] J. Brouckaert et al., "Planer Concave Grating Demultiplexer with High Reflective Bragg Reflector Facets," Photonics Technology Letters, vol. 20, pp. 309-311, 2008. 


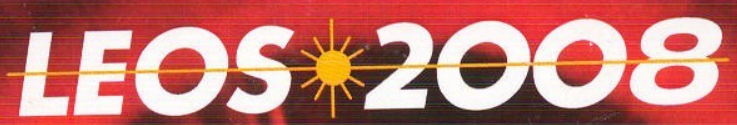

\section{IEEE LEOS Annual Meeting} Conference Proceedings

\section{9 - 13 November 2008 Marriot Newport Beach Hotel \& Spa Nerport Beach, CA}

